\title{
Beamforming with Imperfect CSI
}

\author{
Ye (Geoffrey) $\mathrm{Li}^{*}$ \\ School of Electrical and Computer Engineering \\ Georgia Institute of Technology, Georgia, USA \\ Anthony C. K. Soong, Yinggang Du, and Jianmin Lu \\ Huawei Technologies, Inc. \\ Huawei North American Division, Texas, USA
}

\begin{abstract}
With channel state information (CSI) at the transmitter, beamforming can be used for spatial diversity and multiple spatial access. Due to latency and feedback bandwidth limitation, the CSI at the transmitter is often known with some ambiguity. In this paper, we develop a robust method for downlink beamforming that takes the ambiguity of the CSI into consideration. It is shown by computer simulation that, compared with the existing method, the required signal-to-noise ratio (SNR) for a $1 \%$ bit-error rate (BER) is reduced by over $2 \mathrm{~dB}$ for a system with 4 transmit antennas and 2 users when the variance of the CSI is $-20 \mathrm{~dB}$. The performance gain increases with the number of transmit antennas when the number of users is fixed. The required SNR for a $1 \%$ BER is reduced by over $4 \mathrm{~dB}$ if the the number of transmitter antennas is 8. We also study the impact of power allocation on the downlink beamforming.
\end{abstract}

Key Words: beamforming, partial CSI, transmitter diversity, spatial multiplexing.

\section{INTRODUCTION}

Multiple antenna techniques has emerged as one of the most significant enabling technologies in beyond $3 \mathrm{G}$ wireless communication systems. In these systems, multiple antennas can be mounted at the base station for performance and capacity improvement. However, only a few antennas can be used at the mobile stations because of their size and power consumption limitations. In this case, downlink beamforming can be used for spatial diversity and multiplexing provided that certain channel state information (CSI) is available at the base station.

In the past several years, downlink transmit beamforming has been investigated for spatial diversity and multiplexing, which has been summarized in [1], [2]. In [2], the authors have focused on the capacity improvement of downlink beamforming when different types of CSI statistics are available at the base station. In [3], [4], [5], optimal downlink beamforming has been studied for users with different requirements of signal-to-interference-plus-noise ratio (SINR) when exact channel statistics are known to the transmitter. A multi-user beamforming scheme to maximize signal-to-leakage ratios (SLR) [6] has been proposed when exact CSI is available at the transmitter. Due to channel estimation error, quantization

* Ye (Geoffrey) Li is also holding visiting chair professorship at Communications Laboratory at UESTC, Chengdu, China. The work was done when he consulted YGL Telecomm Lab., Inc. on the MIMO wireless communication project supported by Huawei Technologies, Inc. error (for low data-rate feedback), and delay in feedback, the CSI or channel statistics available at the transmitter for beamforming are imperfect. For transmitter with corrupted channel statistics (co-variance matrix), robust beamforming [8] and optimal power allocation [7] have be proposed.

In this paper, we focus on robust downlink beamforming when imperfect CSI is available at the base station. The rest of this paper is organized as follows. After formulating robust downlink beamforming problem in Section 2, we present our schemes for downlink beamforming and power allocation for users with only one antenna when imperfect CSI is available at the base station in Section 3. Then we present computer simulation results in Section 4. The paper is concluded in Section 5.

\section{SySTEM DESCRIPTION}

Figure 1 shows a down-link cellular system with spatial multiplexing and diversity. In the system, there are $N_{T}$ transmit antennas at the base station and $K\left(K \leq N_{T}\right)$ active users, each with one receive antenna, sharing the same frequency band by means of spatial division multiple access. Without loss of generality, the fading of the channel is assumed to be flat and the CSI is fed back to the base station for beamforming. Denote $h_{i k}$ as the (complex) channel gain corresponding to the $i$-th transmit antenna at the base station and the $k$-th user. Furthermore, we assume that $h_{i k}$ 's are (circular) complex Gaussian with zero mean and unit variance and are independent for different $i$ 's or $k$ 's. The CSI estimated at the receiver is fed back to the base station. Due to channel estimation error, quantization error (for low data rate feedback), and delay in feedback, the CSI available at the base station for beamforming is imperfect and can be expressed as

$$
\hat{h}_{i k}=h_{i k}+e_{i k},
$$

where $e_{i k}$ is the error in the CSI, which is assumed to be complex Gaussian with zero mean and variance $\sigma_{h}^{2}$, independent of $h_{i k}$, and independent and identically distributed (i.i.d.) for different $i$ 's and $k$ 's. Denote

$\hat{\mathbf{h}}_{k}=\left(\begin{array}{c}\hat{h}_{1 k} \\ \vdots \\ \hat{h}_{N_{T} k}\end{array}\right), \mathbf{h}_{k}=\left(\begin{array}{c}h_{1 k} \\ \vdots \\ h_{N_{T} k}\end{array}\right)$, and $\mathbf{e}_{k}=\left(\begin{array}{c}e_{1 k} \\ \vdots \\ e_{N_{T} k}\end{array}\right)$

Then,

$$
\hat{\mathbf{h}}_{k}=\mathbf{h}_{k}+\mathbf{e}_{k} .
$$




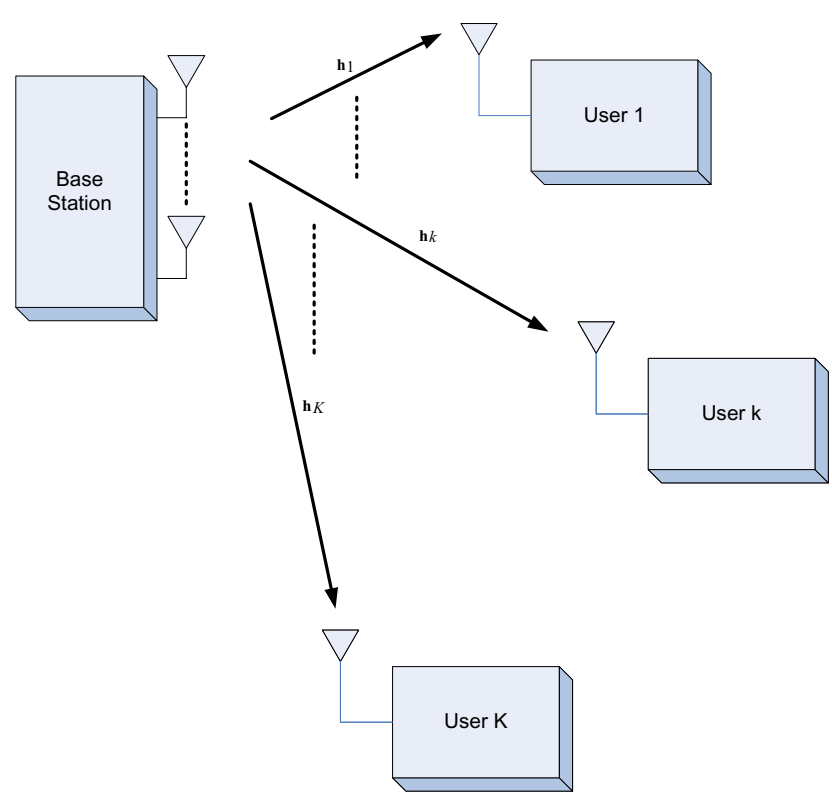

Fig. 1. Beamforming with partial CSI.

With imperfect CSI at the base station, a beam is formed for each user for spatial diversity. Denote $\mathbf{u}_{k}=$ $\left(u_{1 k}, \cdots, u_{N_{T} k}\right)^{T}$ the steering vector for the $k$-th user. The transmitted signal vector at the base station can be expressed as

$$
\mathbf{x}=\sum_{k=1}^{K} \mathbf{u}_{k} s_{k}
$$

where $s_{k}$ is the transmitted symbol for the $k$-th user, which is assumed to be i.i.d. and with zero mean and unit variance. The received signal at the $k$-th user will be

$$
r_{k}=\underbrace{\mathbf{h}_{k}^{T} \mathbf{u}_{k} s_{k}}_{\text {desired signal }}+\underbrace{\sum_{l \neq k} \mathbf{h}_{k}^{T} \mathbf{u}_{l} s_{l}}_{\text {MUI }}+\underbrace{n_{k}}_{\text {AWGN }},
$$

where the first term is the desired signal component, $\sum_{l \neq k} \mathbf{h}_{l}^{T} \mathbf{u}_{k} s_{l}$ is the multi-user interference (MUI), and $n_{k}$ is the additive white Gaussian noise (AWGN) at the $k$-th user, which is assumed to be with zero mean and variance $\sigma_{n}^{2}$.

From the received signal in (3), the instantaneous SINR will be

$$
\gamma_{k}=\frac{\left|\mathbf{h}_{k}^{T} \mathbf{u}_{k}\right|^{2}}{\sum_{l \neq k}\left|\mathbf{h}_{k}^{T} \mathbf{u}_{l}\right|^{2}+\sigma_{n}^{2}} .
$$

\section{BEAMFORMING WITH IMPERFECT CSI}

In this section, we discuss beamforming with imperfect CSI and investigate power allocation for further performance improvement.

\section{A. Beamforming}

At the base station, only imperfect CSI, $\hat{h}_{i k}$ is observed. The observed signal component for user $k$ will, therefore, be

$$
\mathbf{h}_{k}^{T} \mathbf{u}_{k}=\left(\hat{\mathbf{h}}_{k}-\mathbf{e}_{k}\right)^{T} \mathbf{u}_{k},
$$

if the steering vector, $\mathbf{u}_{k}$, is used. Consequently, given imperfect CSI at the base station, the average power of the desired signal component of user $k$ is

$$
\begin{aligned}
\lambda_{k k} & =E\left|\left(\hat{\mathbf{h}}_{k}-\mathbf{e}_{k}\right)^{T} \mathbf{u}_{k}\right|^{2} \\
& =\left|\hat{\mathbf{h}}_{k}^{T} \mathbf{u}_{k}\right|^{2}+\sigma_{h}^{2}\left\|\mathbf{u}_{k}\right\|^{2} .
\end{aligned}
$$

Similarly, the interference power at the $k$-th user from the transmitted symbol for the $n$-th user can be found to be

$$
\lambda_{n k}=\left|\hat{\mathbf{h}}_{k}^{T} \mathbf{u}_{n}\right|^{2}+\sigma_{h}^{2}\left\|\mathbf{u}_{n}\right\|^{2} .
$$

Ideally, we should select the steering vectors, $\mathbf{u}_{k}$ for $k=$ $1, \cdots, K$, to maximize the minimum of the observed SINR at the transmitter; that is, choose $\mathbf{u}_{k}$ for $k=1, \cdots, K$ to maximize

$$
\gamma\left(\mathbf{u}_{1}, \cdots, \mathbf{u}_{K}\right)=\min _{1 \leq k \leq K}\left\{\frac{\lambda_{k k}}{\sum_{l \neq k} \lambda_{l k}+\sigma_{n}^{2}}\right\} .
$$

However, the max-min or min-max problem is usually untractable and has no closed form solution. The optimal solution can, therefore, only be obtained by iteration [5], which is usually computationally complicated.

Instead of dealing with the max-min problem, we choose each steering vector to maximize the ratio of the signal-toleakage ratio(SLR), as in [6], that is,

$$
\gamma_{k}\left(\mathbf{u}_{k}\right)=\frac{\lambda_{k k}}{\sum_{l \neq k} \lambda_{k l}}
$$

Using (5) and (6), (7) can be further expressed as

$$
\begin{aligned}
\gamma_{k}\left(\mathbf{u}_{k}\right) & =\frac{\left|\hat{\mathbf{h}}_{k}^{T} \mathbf{u}_{k}\right|^{2}+\sigma_{h}^{2}\left\|\mathbf{u}_{k}\right\|^{2}}{\sum_{l \neq k}\left(\left|\hat{\mathbf{h}}_{l}^{T} \mathbf{u}_{k}\right|^{2}+\sigma_{h}^{2}\left\|\mathbf{u}_{k}\right\|^{2}\right)} \\
& =\frac{\mathbf{u}_{k}^{H} \mathbf{R}_{s k} \mathbf{u}_{k}}{\mathbf{u}_{k}^{H} \mathbf{R}_{i k} \mathbf{u}_{k}}
\end{aligned}
$$

where

$$
\mathbf{R}_{s k}=\hat{\mathbf{h}}_{k}^{*} \hat{\mathbf{h}}_{k}^{T}+\sigma_{h}^{2} \mathbf{I}
$$

and

$$
\mathbf{R}_{i k}=\sum_{l \neq k} \hat{\mathbf{h}}_{l}^{*} \hat{\mathbf{h}}_{l}^{T}+(K-1) \sigma_{h}^{2} \mathbf{I}
$$

It can be easily seen that both $\mathbf{R}_{s k}$ and $\mathbf{R}_{i k}$ are positive definite if $\sigma_{h}^{2} \neq 0$. Let the eigen-decomposition of $\mathbf{R}_{i k}$ be

$$
\mathbf{R}_{i k}=\mathbf{U}_{k}\left(\begin{array}{cccc}
d_{1 k}^{2} & 0 & \cdots & 0 \\
0 & d_{2 k}^{2} & \ddots & 0 \\
\vdots & \ddots & \ddots & \vdots \\
0 & 0 & \cdots & d_{K k}^{2}
\end{array}\right) \mathbf{U}_{k}^{H},
$$

where $d_{l k}$ are all positive and $\mathbf{U}_{k}$ is a unitary matrix. Denote

$$
\mathbf{v}_{k}=\mathbf{D}_{k} \mathbf{U}_{k}^{H} \mathbf{u}_{k}
$$

or

$$
\mathbf{u}_{k}=\mathbf{U}_{k} \mathbf{D}_{k}^{-1} \mathbf{v}_{k},
$$


where

$$
\mathbf{D}_{k}=\left(\begin{array}{cccc}
d_{1 k} & 0 & \cdots & 0 \\
0 & d_{2 k} & \ddots & 0 \\
\vdots & \ddots & \ddots & \vdots \\
0 & 0 & \cdots & d_{K k}
\end{array}\right)
$$

and

$$
\mathbf{D}_{k}^{-1}=\left(\begin{array}{cccc}
d_{1 k}^{-1} & 0 & \cdots & 0 \\
0 & d_{2 k}^{-1} & \ddots & 0 \\
\vdots & \ddots & \ddots & \vdots \\
0 & 0 & \cdots & d_{K k}^{-1}
\end{array}\right)
$$

Then

$$
\gamma_{k}\left(\mathbf{u}_{k}\right)=\frac{\mathbf{v}_{k}^{H} \mathbf{D}_{k}^{-1} \mathbf{U}_{k}^{H} \mathbf{R}_{s k} \mathbf{U}_{k} \mathbf{D}_{k}^{-1} \mathbf{v}_{k}}{\left\|\mathbf{v}_{k}\right\|^{2}}
$$

Let $\gamma_{o k}$ be the largest eigen-value of $\mathbf{D}_{k}^{-1} \mathbf{U}_{k}^{H} \mathbf{R}_{s k} \mathbf{U}_{k} \mathbf{D}_{k}^{-1}$ and $\mathbf{v}_{o k}$ be the corresponding eigen-vector. Then, $\gamma_{k}\left(\mathbf{u}_{k}\right)$ reaches its maximum value, $\gamma_{o k}$, when $\mathbf{u}_{k}$ takes

$$
\mathbf{u}_{o k}=\mathbf{U}_{k} \mathbf{D}_{k}^{-1} \mathbf{v}_{o k} \text {. }
$$

Usually, the vector calculated from (9) is not necessarily normalized. Steering vector can be obtained by normalizing $\mathbf{u}_{o k}$.

\section{B. Optimum Power Allocation}

In the previous section, we have discussed steering vector optimization for each user to maximize the ratio of the desired signal power and the leakage power (the overall power of interference to other users). In this section, we will discuss transmission power allocation among active users to further optimize the whole system. Without loss of generality, we assume that the steering vectors obtained in the previous section has already been normalized, that is, $\left\|\mathbf{u}_{o k}\right\|=1$.

There are two different ways to optimize the power allocation. We can minimize the total transmission power for a given SINR constraint of each user [5], [7]. Alternatively, we can also maximize the SINR for a given total transmission power of all active users. We will focus on the later one here.

Assume that the average transmission power of each user is unit. Then for a system with $K$ active users, the total transmission power will be $K$. Let the transmission power for user $k$ be $p_{k}$. Then

$$
\sum_{k=1}^{K} p_{k}=K \text { and } p_{k}>0 .
$$

As before, denote $\lambda_{n k}$ be the power of interference/desired signal when the optimum steering vector is used. Then the SINR for the $k$-th user will be

$$
\gamma_{k}\left(p_{1}, \cdots, p_{K}\right)=\frac{p_{k} \lambda_{k k}}{\sum_{l \neq k} p_{l} \lambda_{l k}+\sigma_{n}^{2}} .
$$

It can be easily seen that we can always adjust the power distribution to increase the lower $\gamma_{k}\left(p_{1}, \cdots, p_{K}\right)$ as long as they are not equal. As a result, all $\gamma_{k}\left(p_{o 1}, \cdots, p_{o K}\right)$ 's must be equal for optimal power allocation. Therefore, the optimal SINR, $\gamma_{o}$, and power allocation can be obtained by the following identities

$$
p_{o k} \lambda_{k k}=\gamma_{o} \sum_{l \neq k} p_{o l} \lambda_{l k}+\gamma_{o} \sigma_{n}^{2},
$$

for $k=1, \cdots, K$ and

$$
\sum_{k=1}^{K} p_{o k}=K .
$$

Denote

$$
\begin{gathered}
\boldsymbol{\Lambda}_{d}=\operatorname{diag}\left\{\lambda_{11}, \cdots, \lambda_{K K}\right\} \\
\boldsymbol{\Lambda}=\left(\begin{array}{ccc}
\lambda_{11} & \cdots & \lambda_{K 1} \\
\vdots & \cdots & \vdots \\
\lambda_{1 K} & \cdots & \lambda_{K K}
\end{array}\right),
\end{gathered}
$$

and the optimal power allocation vector

$$
\mathbf{p}_{o}=\left(p_{o 1}, \cdots, p_{o K}\right)^{T} .
$$

Then (10) can be expressed into a more compact form as

$$
\left(\left(1+\frac{1}{\gamma_{o}}\right) \boldsymbol{\Lambda}_{d}-\boldsymbol{\Lambda}\right) \mathbf{p}_{o}=\sigma_{n}^{2} \mathbf{1},
$$

and

$$
\mathbf{1}^{T} \mathbf{p}_{o}=K,
$$

where

$$
\mathbf{1}=(\underbrace{1, \cdots, 1}_{\mathrm{K} 1 \text { 's }})^{T} \text {. }
$$

From (11),

$$
\mathbf{p}_{o}=\sigma_{n}^{2}\left(\left(1+\frac{1}{\gamma_{o}}\right) \boldsymbol{\Lambda}_{d}-\boldsymbol{\Lambda}\right)^{-1} \mathbf{1} .
$$

Then the optimal SNIR $\left(\gamma_{o}\right)$ is determined by the following identity

$$
\sigma_{n}^{2} \mathbf{1}^{T}\left(\left(1+\frac{1}{\gamma_{o}}\right) \boldsymbol{\Lambda}_{d}-\boldsymbol{\Lambda}\right)^{-1} \mathbf{1}=K .
$$

Once $\gamma_{o}$ is determined by (13), the optimal power allocation can be found from (12).

When system is interference limited, that is, the signalto-noise ratio is very large, or $\sigma_{n}^{2} \approx 0$, the optimum power allocation approach can be simplified. In that case, denote

$$
\tilde{\mathbf{p}}_{o}=\boldsymbol{\Lambda}_{d} \mathbf{p}_{o}
$$

and

$$
\tilde{\Lambda}=\boldsymbol{\Lambda} \Lambda_{d}^{-1} .
$$

Then (11) turns into

$$
\left(1+\frac{1}{\gamma_{o}}\right) \tilde{\mathbf{p}}_{o}=\tilde{\boldsymbol{\Lambda}} \tilde{\mathbf{p}}_{o} .
$$

Note that $\tilde{\Lambda}$ is a non-negative matrix. From [3], [9], there is an nonnegative vector, $\tilde{\mathbf{p}}_{o}$, with $\tilde{p}_{o k} \geq 0$ for $k=1, \cdots, K$ such that

$$
\rho \tilde{\mathbf{p}}_{o}=\tilde{\boldsymbol{\Lambda}} \tilde{\mathbf{p}}_{o} .
$$

Then the optimum power allocation will be

$$
\mathbf{p}_{o}=\boldsymbol{\Lambda}_{d}^{-1} \tilde{\mathbf{p}}_{o},
$$


and the optimum signal-to-interference ratio (SIR) in this case will be

$$
\gamma_{o}=\frac{1}{\rho-1} .
$$

\section{Simulation Results}

In this section, we demonstrate the performance of the proposed algorithm by computer simulation. In our simulation, channel gains corresponding to different pairs of transmit and receive antennas, $h_{i k}$ 's, are assumed to be independent, complex (circular) Gaussian with zero mean and unit variance. Only imperfect CSI is available at the transmitter for downlink beamforming. The transmitted symbols are independent and are randomly drawn from 4-QAM constellations, $\left\{ \pm \frac{1}{\sqrt{2}} \pm\right.$ $\left.\jmath \frac{1}{\sqrt{2}}\right\}$, each with the same probability.
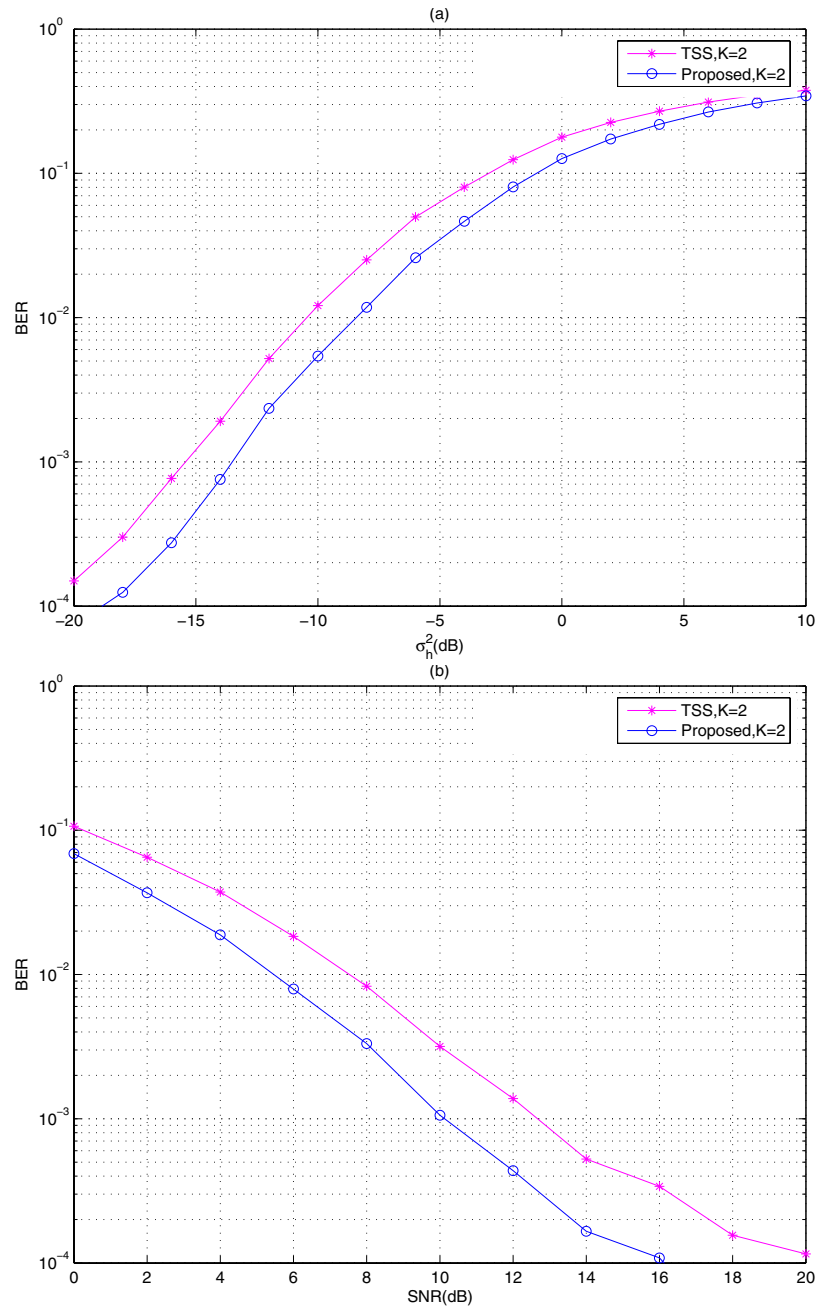

Fig. 2. Performance of downlink beamforming for a system with $N_{T}=4$ transmit antennas and $K=2$ users, (a) BER versus CSI ambiguity when $\mathrm{SNR}=20 \mathrm{~dB}$ and (b) BER versus SNR when $\sigma_{h}=-20 \mathrm{~dB}$.

\section{A. Beamforming with Imperfect CSI}

Figures 2-4 compare the performance of the proposed method with the one in [6] for systems with different numbers of transmit antennas and users. From those figures, we can see that the proposed method is more robust to CSI ambiguity. In particular, from Figure 2, the required SNR for a $1 \%$ BER
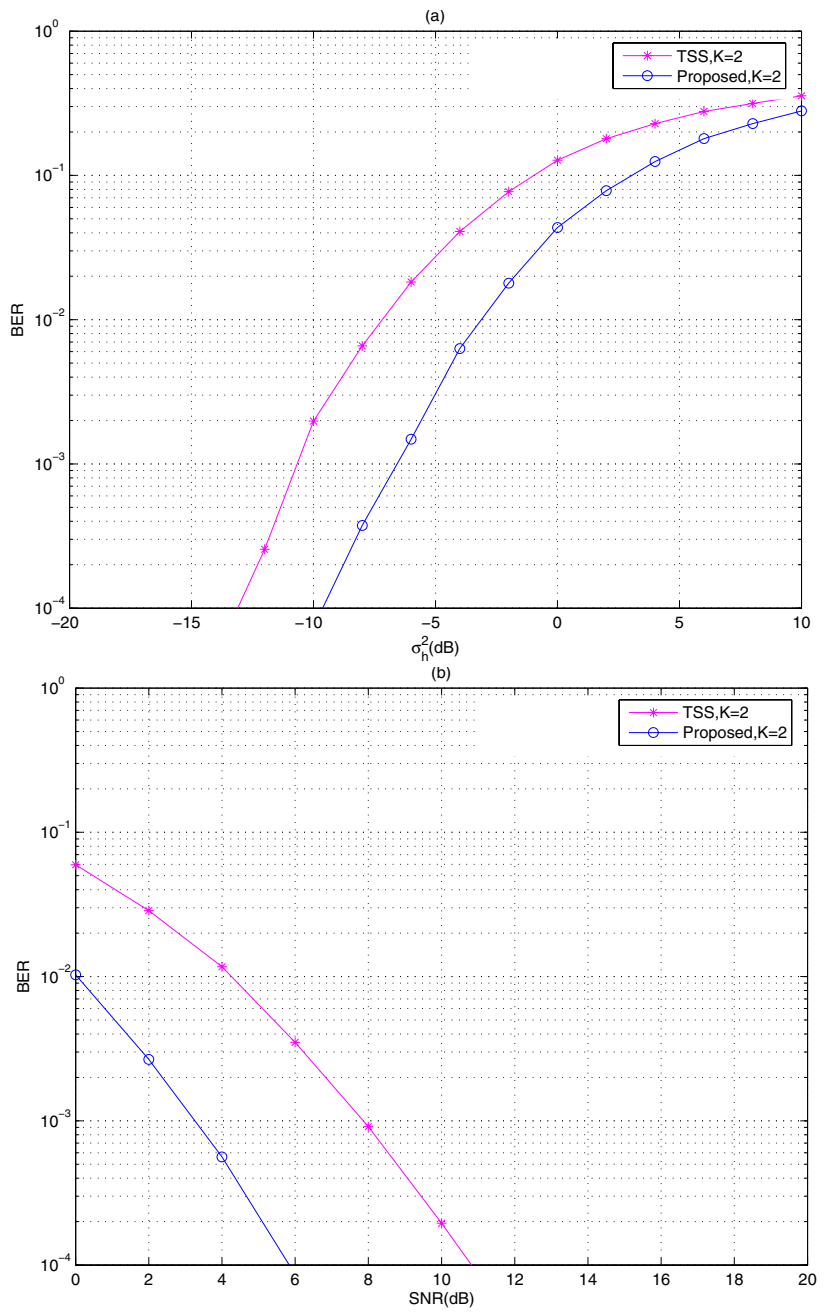

Fig. 3. Performance of downlink beamforming for a system with $N_{T}=8$ transmit antennas and $K=2$ users, (a) BER versus CSI ambiguity when $\mathrm{SNR}=20 \mathrm{~dB}$ and (b) BER versus SNR when $\sigma_{h}=-20 \mathrm{~dB}$.

is reduced by over $2 \mathrm{~dB}$ compared with the algorithm in [6] for a system with $N_{T}=4$ transmit antennas, $K=2$ users, and $\sigma_{h}=-20 \mathrm{~dB}$. The performance gain increases with the number of transmit antennas when the number of users is fixed. As we can see from Figure 3, the required SNR for a $1 \%$ BER is reduced by over $4 \mathrm{~dB}$ if the number of transmit antennas is changed into $N_{T}=8$ in the above environment. If we fix the number of transmit antennas and increase the number of users, then the performance gain will be reduced, as we can see by comparing Figure 3 and Figure 4 .

\section{B. Optimum Power Allocation}

To demonstrate the effectiveness of optimum power allocation, we consider shadowing in wireless channels. In the simulation here, we assume a log-normal shadowing with an $8 \mathrm{~dB}$ standard deviation. The shadowing is same for different transmitter antennas at the same mobile; however, independent for different mobiles. Figure 5 demonstrates the performance improvement through power allocation with imperfect CSI for a system with different numbers of transmit antennas and users when $\sigma_{h}=-20 \mathrm{~dB}$. From the figure, we can see significant performance improvement, especially when the SNR is high. 

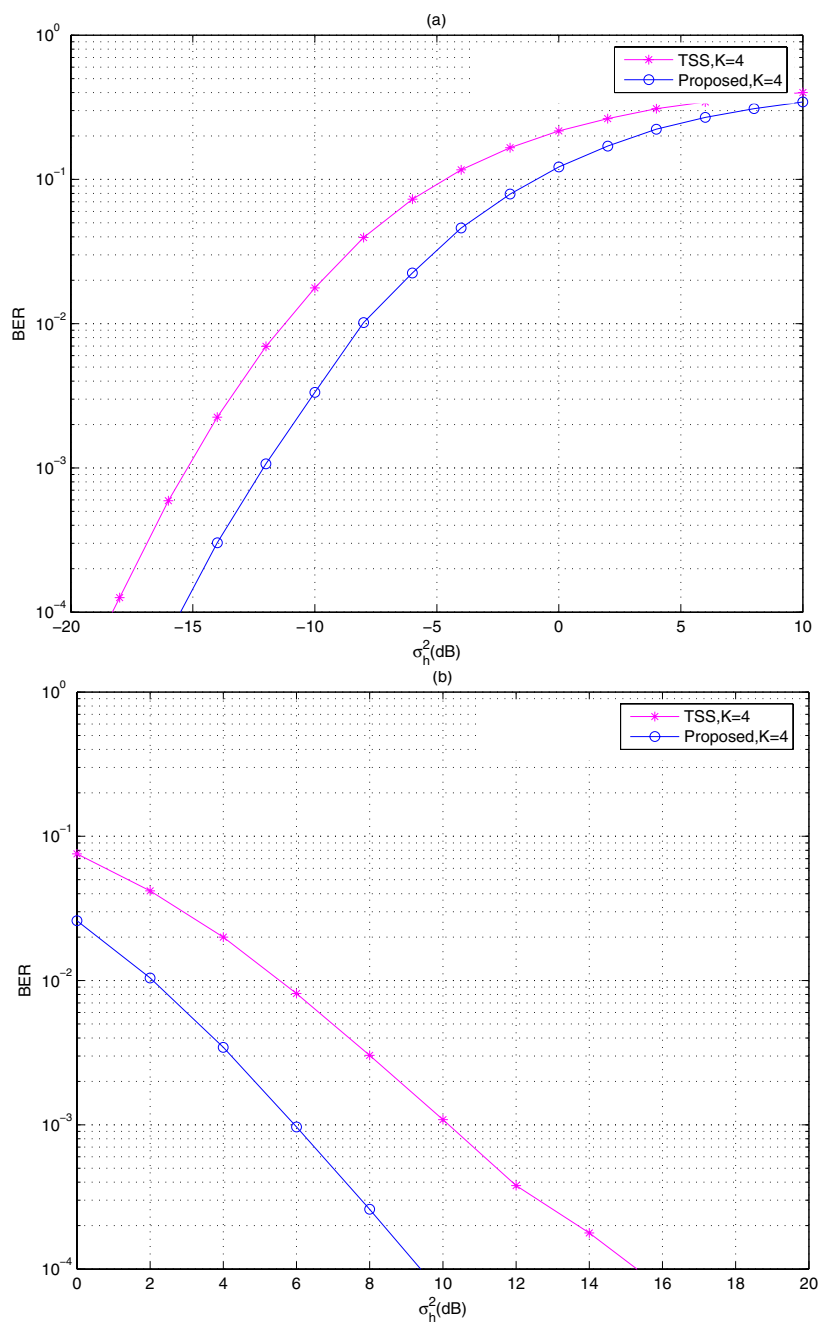

Fig. 4. Performance of downlink beamforming for a system with $N_{T}=8$ transmit antennas and $K=4$ users, (a) BER versus CSI ambiguity when $\mathrm{SNR}=20 \mathrm{~dB}$ and (b) BER versus SNR when $\sigma_{h}=-20 \mathrm{~dB}$.

\section{CONCLUSIONS}

In practical mobile communication systems, the base station can have multiple antennas while the handsets can have at most a couple of antennas. The multiple antennas at the base station can be used for downlink beamforming for spatial diversity and spatial division multiple access. To perform downlink beamforming, CSI is usually required at the transmitter, which is usually obtained through feedback from the receiver. Due to bandwidth limitation and latency, the CSI at the transmitter is usually known with some ambiguity. In this paper, we have proposed a novel method for robust downlink beamforming with imperfect CSI. It is shown by computer simulation that the proposed method can significantly improve the system performance compared with the existing one that does not take CSI ambiguity into consideration in performing beamforming. Therefore, the proposed method is a potential technique for future mobile communications. Even through we have assumed that there is only one receive antenna at the mobile station in developing the algorithms in the paper, with minor modification, the developed algorithms can be also used for the systems with multiple receive antennas.
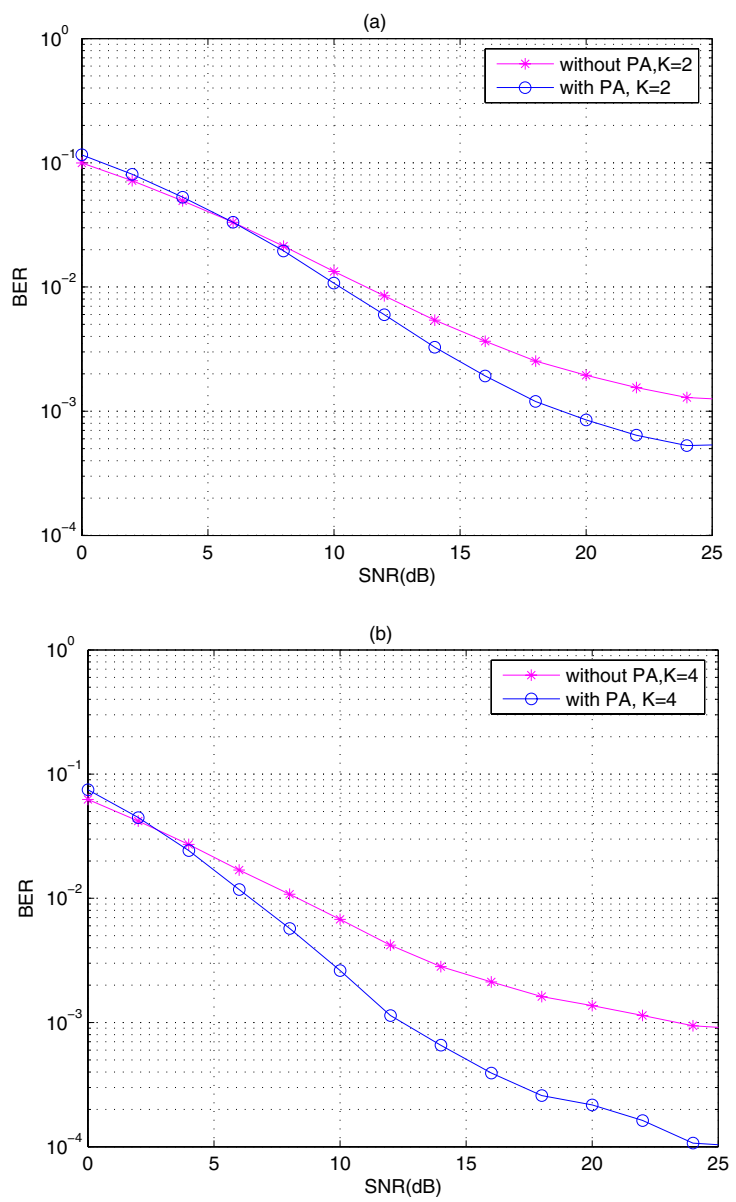

Fig. 5. Impact of adaptive power allocation on a system with (a) $N_{T}=4$ transmit antennas and $K=2$ users, (b) $N_{T}=8$ transmit antennas and $K=4$ users when $\sigma_{h}=-10 \mathrm{~dB}$.

\section{REFERENCES}

[1] Q. H. Spencer, C. B. Peel, A. L. Swindlehurst, and M. Haardt, "An introduction to the multi-user MIMO downlink," IEEE Commun. Mag. pp. 60-67, Oct. 2004.

[2] A. Goldsmith, S. A. Jafar, N. Jindal, and S. Vishwanath, "Capacity limits of MIMO channels," IEEE J. Sel. Areas Commun., vol. 21, no. 5, June 2003.

[3] H. Boche and M. Schubert, "A general duality theory for uplink and downlink beamforming," Proc. of IEEE 55th Veh. Tech. Conf., pp. 19111915, May 2002.

[4] M. Schubert and H. Boche, "Solution of the multiuser downlink beamforming problem with individual SINR constrains," IEEE Trans. Veh. Tech. vol. 53, no. 1, pp. 18-28, Jan. 2004.

[5] M. Schubert and H. Boche, "Iterative multiuser uplink and downlink beamforming under SINR constrains," IEEE Trans. Signal Processing vol. 53, no. 1, pp. 2324-2334, Jan. 2004.

[6] A. Tarighat, M. Sadek, and A. H. Sayed, "A multi user beamforming scheme for downlink MIMO channels based on maximizing signal-toleakage ratios," Proc. of IEEE International Conf. on Acoustics, Speech, and Signal Processing (ICASSP'05), pp. 18-23, March 2005.

[7] M. Biguesh, S. Shahbazpanahi, and A. B. Gershman, "Robust downlink power adjustment in cellular communication systems with antenna arrays at base stations," Proc. of 2003 4th IEEE Workshop on Signal Processing Advances in Wireless Commun., pp. 634-638, June 2003.

[8] B. K. Chalise and A. Czylwik, "Robust downlink beamforming based upon outage probabiltiy criterion," Proc. of IEEE 60th Veh. Tech. Conf., pp. 334-338, Sept. 2004.

[9] R. A. Horn and C. R. Johnson, Matrix Analysis, Cambridge University Press, 1985. 